\title{
PERFORMANCE COMPARISON OF NOISE REDUCTION IN MAMMOGRAM IMAGES
}

\author{
S. Kannan ${ }^{1}$, N. P. Subiramaniyam ${ }^{2}$, A. T. Rajamanickam ${ }^{3}$, A. Balamurugan ${ }^{4}$ \\ ${ }^{1,2,3}$ Assistant Professor, Department of ECS, Nehru Arts and Science College, Tamilnadu, India. 641105 \\ ${ }^{4}$ Assistant Professor, Department of Physics, Government Arts College, Udhagamandalam, Tamilnadu, India. 643002
}

\begin{abstract}
Noise level present in mammogram images highly affects the image analysis and accuracy of classification. Hence removing noise present in mammogram images is an important task. Noise present in the medical images depends on imaging modalities. The dominant noise present in mammogram images are quantum noise. The objective of this work is to study the various filters such as mean, median and wiener filter with different window size using standard benchmark (Digital Database for Screening Mammography) DDSM dataset. Higher the value of the (Peak Signal to Noise Ratio) PSNR, better the image quality of the restored image. The restored image quality of various filters was evaluated with PSNR value. We obtained, the wiener filter with window size $3 \times 3$ gives better result for noise reduction in mammogram images.
\end{abstract}

Key Words: Mammogram, Quantum Noise, Mean Filter, Median Filter, Wiener Filter, DDSM and PSNR

\section{INTRODUCTION}

The imaging noises reduce the quality of the acquired medical images. The noise present in the images are depends on the imaging modalities. In X-ray mammogram, the dominant noise is quantum noise. Images cannot be used as they are, because they contain some noise. Hence removal of noise that might result while scanning is important to get accurate information. In the noise removal process, filtering techniques are used. In this paper we are analyzing different filtering technique to find the efficient one in the process of Digital mammogram images enhancement.

\section{MAMMOGRAM IMAGES}

Mammography has been proved to be the primary radiologic procedure for the early detection of breast cancer. The indicator of breast cancer in a mammogram is the presence of a tumor mass. DDSM database images are used for the experimental study. Quantum noise are the dominant in mammogram.

\section{QUANTUM NOISE}

Noise level present in the images highly affect the quality and accuracy of classification when working with Mammographic images. The dominant noise present in the mammographic images are quantum noise. Quantum noise occurs inherently in low - dose $\mathrm{X}$-ray imaging due to the very low $\mathrm{X}$ - ray quantum counts.

\section{DENOISING TECHNIQUES}

Filtering technique are used to denoise the image. In this paper we describe several filtering methods such as mean, median, wiener filter to reduce the quantum noise present in mammogram images.

\subsection{Mean Filter}

Let $S_{x y}$ represent the set of coordinates in the window $\mathrm{m} \mathrm{x} \mathrm{n}$, centered at point $(\mathrm{x}, \mathrm{y})$.The mean filter computes the average of value of the corrupted image $g(x, y)$ in the area defined by $S_{x y}$

$f(x, y)=\frac{1}{m n} \sum_{(s, t) \in S_{x y}} g(s, t)$

where $f(x, y)$ Value of restored image at point $(x, y)$

\subsection{Median Filter}

Here the magnitude of all the vectors is taken with in a mask and arranged according to the magnitude. The median magnitude pixel is then used to replace the pixel studied. This filter is given by

Median filter $\left(X_{1}, X_{n}\right)=$ median $\left(\Pi x_{1} \Pi_{2} \ldots \Pi x_{N} \Pi_{2}\right)$

\subsection{Wiener Filter}

The following are the characteristics of the wiener filter.

a. Signal and noise are stationary. The linear random proces are made with known spectral characteristics.

b. Requirement: Filter must be physically realizable.

c. Performance criteria:

$$
G(u, v)=\frac{H^{*}(u, v) p_{s}(u, v)}{I H(u, v) I 2 p_{s}(u, v)+p_{n}(u, v)}
$$

Where $\mathrm{H}(\mathrm{u}, \mathrm{v})$ is the Degradation Function

$H^{*}(u, v)$ is the complex conjugate of degradation function 
$\mathrm{P}_{\mathrm{n}}(\mathrm{u}, \mathrm{v})$ is the Power spectral density of noise

$\mathrm{P}_{\mathrm{s}}(\mathrm{u}, \mathrm{v})$ is the Power spectral density of undegraded image.

\section{EXPERIMENTAL RESULTS}

The images for this study were obtained from DDSM Database. The performance of the proposed filters is uniformly by resizing all the images to $256 \times 256$ pixels. To evaluate the performance of the various filter, we used PSNR image quality performance metric. The PSNR value of the denoised image is calculated using the following formula.

$$
\text { PSNR }=10 \log _{10} \frac{\sum I \sum i 255^{2}}{\sum I \sum i\left(S_{i, j}-K_{i, j}\right)^{2}} d b
$$

Where $S_{i, j}$ is the original image

$$
\mathrm{K}_{\mathrm{i}, \mathrm{j}} \text { is the restored image. }
$$

Following figure1 shows the input and output image for image 01

\section{DDSM IMAGE 01}

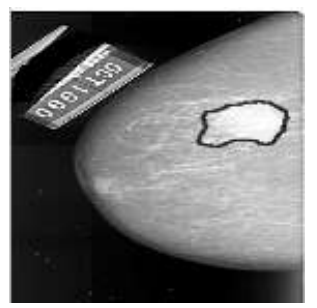

Input image

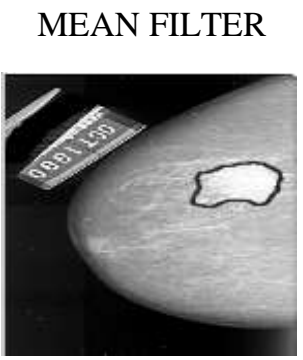

MEDIAN FILTER

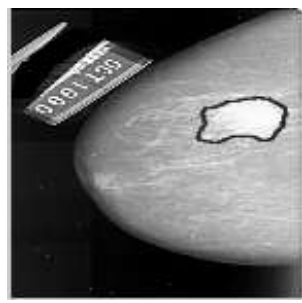

WIENER FILTER

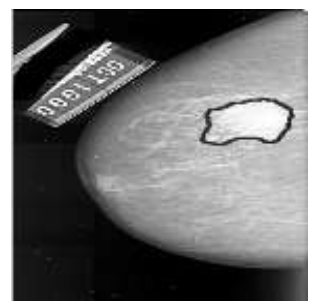

Output image of various filters for Image 01 (window size $3 \times 3$ )

Fig-1: Input and Output Image for Image 01
Table-1: PSNR value of the images studied with different window size and result

\begin{tabular}{|c|c|c|c|c|}
\hline $\begin{array}{c}\text { DDSM } \\
\text { Image }\end{array}$ & $\begin{array}{c}\text { Window } \\
\text { Size }\end{array}$ & $\begin{array}{c}\text { Mean } \\
\text { Filter }\end{array}$ & $\begin{array}{c}\text { Median } \\
\text { Filter }\end{array}$ & $\begin{array}{c}\text { Wiener } \\
\text { Filter }\end{array}$ \\
\hline & $3 \times 3$ & 25.08 & 30.69 & $\mathbf{3 5 . 6 9}$ \\
Image & $5 \times 5$ & 21.68 & 23.94 & 32.40 \\
$\mathbf{0 1}$ & $7 \times 7$ & 20.16 & 22.51 & 30.78 \\
& & & & \\
\hline & $3 \times 3$ & 27.74 & 31.80 & $\mathbf{3 5 . 9 2}$ \\
Image & $5 \times 5$ & 23.87 & 26.62 & 32.35 \\
$\mathbf{0 2}$ & $7 \times 7$ & 21.91 & 23.93 & 30.73 \\
& & & & \\
\hline & $3 \times 3$ & 27.89 & 33.50 & $\mathbf{3 5 . 8 8}$ \\
Image & $5 \times 5$ & 24.17 & 28.30 & 32.57 \\
$\mathbf{0 3}$ & $7 \times 7$ & 22.18 & 22.60 & 31.08 \\
& & & & \\
\hline & $3 \times 3$ & 27.31 & 31.54 & $\mathbf{3 6 . 9 4}$ \\
Image & $5 \times 5$ & 23.31 & 24.85 & 33.08 \\
$\mathbf{0 4}$ & $7 \times 7$ & 21.24 & 20.66 & 31.47 \\
& & & & \\
\hline & $3 \times 3$ & 25.77 & 28.45 & $\mathbf{3 7 . 3 6}$ \\
Image & $5 \times 5$ & 22.70 & 25.19 & 33.72 \\
$\mathbf{0 5}$ & $7 \times 7$ & 21.08 & 23.67 & 31.91 \\
& & & & \\
\hline
\end{tabular}

Following figure2 shows the comparison of PSNR value for image 01 with different window

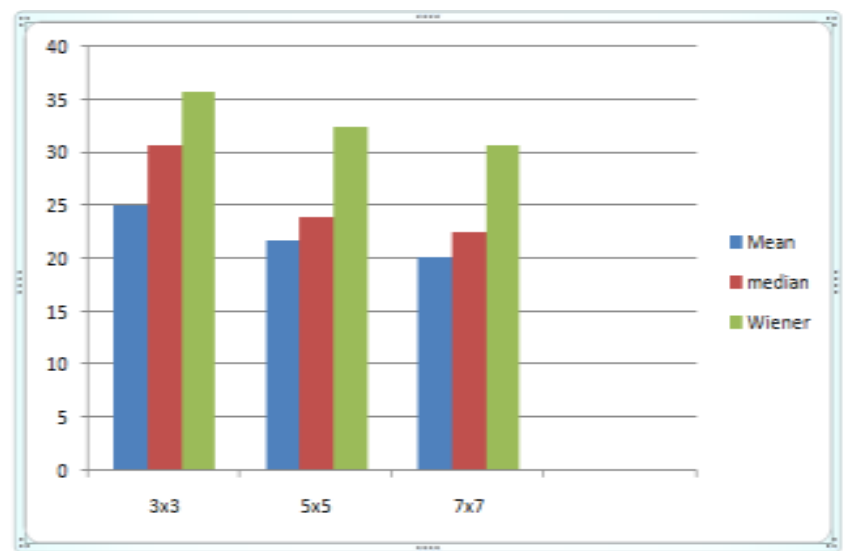

Fig-2: Comparison of PSNR value for Image 01 with Different Window

\section{CONCLUSION}

In this paper, we have presented the performance comparison of mammogram images with different window size using mean, median, wiener filter. The performances are analyzed according to PSNR value. We obtained, the wiener filter with window size $3 \times 3$ gives better result for noise reduction in mammogram images. For future work we would like develop a noise reduction method by combining filters, and also to compare with various image quality evaluation metrics. 


\section{REFERENCES}

[1] R.C. Gonzalez and R.E woods, Digital Image Processing, prentice Hall second ed,2002.

[2] http://marathan.csee.uff.edu/mammography/Database.ht $\mathrm{ml}$

[3] Dr.K.Meenakshi Sundaram,D.Sasikala,P.Aarthi Rani:a Study on Preprocessing a Mammogram Image using Adaptive Median Filter,IJIRSET,Vol 3 ,pp.1033310337,(2014).

[4] Suresh Kumar, Papendra Kumar, Manoj Gupta, Ashok Kumar Nagwat,:Performance comparison of median and wiener filter in Image De-noising, International journal of computer Applications, volume 12, pp. 0975 $-8887,(2010)$.

[5] Cheng H.D., Xiaopeng Cai, Xiaowei Chen, Liming Hu, Xueling Lou,: Computer-aided detection and classification of micro calcifications in mammograms: A survey, Pattern Recogn. Vol 36 , pp. 2967-2991, (2003)

[6] Ioanna Christoyiani, Evalgelos Dermatas, George Kokkinakis,: Fast Detection of Masses in Computer Aided Mammography, IEEE Signal Processing Magazine, pp: 54-64, (2000). 